\title{
Analytical Model for High Level Power Modeling of Combinational and Sequential Circuits ${ }^{\dagger}$
}

\author{
Subodh Gupta and Farid N. Najm \\ ECE Dept. and Coordinated Science Lab. \\ University of Illinois at Urbana-Champaign \\ Urbana, Illinois 61801
}

\begin{abstract}
In this paper, we propose a modeling approach that captures the dependence of the power dissipation of a (combinational or sequential) logic circuit on its input/output signal switching statistics. The resulting power macromodel, consists of a quadratic or cubic equation in four variables, that can be used to estimate the power consumed in the circuit for any given input/output signal statistics. Given a low-level (typically gate-level) description of the circuit, we describe a characterization process that uses a recursive least squares $(R L S)$ algorithm by which such a equation-based model can be automatically built. The four variables of our model are the average input signal probability, average input switching activity, average input spatial correlation coefficient and average output zero-delay switching activity. This approach has been implemented and models have been built and tested for many combinational and sequential benchmark circuits.
\end{abstract}

\section{Introduction}

With the advent of portable and high-density micro-electronic devices, the power dissipation of very large scale integrated (VLSI) circuits has become a critical concern. Modern microprocessors are hot, and their power consumption can exceed 30 or 50 Watts. Due to limited battery life, reliability issues, and packaging/cooling costs, power consumption has become a more critical design concern than speed and area in some applications. Hence to avoid problems associated with excessive power consumption, there is a need for CAD tools to help in estimating the power consumption of VLSI designs.

A number of CAD techniques have been proposed for gate-level power estimation (see [1] for a survey). However, by the time the design has been specified down to the gate level, it may be too late or too expensive to go back and fix high power problems. Hence in order to avoid costly redesign steps, power estimation tools are required that can estimate the power consumption at a high level of abstraction, such as when the circuit is represented only by the Boolean equations. This will provide the designer with more flexibility to explore design trade-offs early in the design process, reducing the design cost and time.

In response to this need, a number of high-level power estimation techniques have been recently proposed (see [2] for a survey). Two styles of techniques have been proposed, which we refer to as top-down and bottom-up. In the top-down techniques $[3,4]$, a combinational circuit is specified only as a Boolean function, with no information on the circuit structure, number of gates/nodes, etc. Top-down methods are useful when one is designing a logic block that was not previously designed, so that its internal details are unknown.

In contrast, bottom-up methods [5-11] are useful when one is reusing a previously-designed logic block, so that all the internal structural details of the circuit are known. In this case, one develops a power macromodel for this block which can be used during high-level power estimation (of the overall system in which this block is used), in order to estimate the power dissipation of this block without performing a more expensive gate-level power estimation on it.

The method in [5] uses the power factor approximation technique, which treats all the circuit input bits as digital "white noise" and due to this assumption can give errors of up to $80 \%$ in comparison to gate-level tools. Although [6] gives more accurate result, its main disadvantage is that it treats different modules differently, requiring specialized analytical expressions for the power to be provided by the user. Thus, depending upon the functionality of the module, a different type of macromodel (analytical equation) may have to be used.

The method in [7] characterizes the power dissipation of circuits based on input transitions rather than input statistics. Since the number of possible input transitions for an $n$-input combinational circuit is $2^{2 n}$, they present a clustering algorithm to compress the input transitions into clusters of input transitions that have the same power values (approximately). They use

$\dagger$ This research was supported in part by the National Science Foundation (NSF MIP 97-10235) and by the Semiconductor Research Corporation (SRC 97-DJ-484), with technical mentorship from Texas Instruments Inc. 
heuristics to implement the clustering algorithm, but it is not clear how efficient the method would be on large circuits.

In [8], the authors present a technique to estimate switching activity and power consumption at the RTL for data path and control circuits, in the presence of glitching activity. To construct a power macromodel, they use both analytical equations and look-up tables. The method is quite good and uses 9 or more variables in the power macromodel. Our independent work has shown that it is possible to construct a look-up table power macromodel with much fewer variables (4 can be enough).

In [9], the authors presented a macromodel for estimating the cycle-by-cycle power at the RTL. The proposed methodology consists of three steps: module equation form generation and variable selection, variable reduction, and population stratifications. The generated macromodel has 15 variables. They show good accuracy in estimating average and cycle-by-cycle power. The macromodels are dependent on a training vector set, so that the accuracy is compromised if the training set is not similar to the vector set to be applied.

All the approaches discussed above are limited to only combinational circuits. In this paper, we propose a power macromodeling approach for both combinational and sequential circuits that (1) takes into account the effect of the circuit input switching activity and does not treat the circuit inputs as white noise, (2) takes into account input correlation, both spatial and temporal and (3) is based on a single fixed macromodel template which does not depend on the type of circuit being analyzed. Our model is equation-based. Specifically, we construct a quadratic or cubic equation in the following four variables: average input signal probability $\left(P_{\text {in }}\right)$, average input switching activity $\left(D_{i n}\right)$, average input spatial correlation coefficient $\left(S C_{i n}\right)$, and average output zero delay switching activity $\left(D_{\text {out }}\right)$. For a logic node, the switching activity, also called the transition density [12], is defined as the average number of logic transitions per unit time. The zero delay switching activity refers to the case when the circuit gates are considered to have zero delay, so that only truly required logic transitions (and no hazards or glitches) are observed. From a high-level view, it is reasonable to assume that fast functional simulation will be applied to measure signal switching statistics, so that only the zero delay output activity (and not the real delay output activity) will be computed. The main advantage of our approach is that all types of circuits are treated in the same way, i.e., we do not use different model equation types for different modules. As a result, the method is very easy to use, and requires no user intervention. Indeed, we will present an automatic characterization procedure by which the macromodel can be built for a given circuit.

This paper is organized as follows. In section 2, we will give some background regarding our original 4-dimensional (4D) table-based macromodel. In section 3, we will explain why the model holds just as well for sequential circuits. In section 4, we discuss the new analytical-equationbased formulation of the $4 \mathrm{D}$ model and describe the characterization procedure. In section 5 , we give empirical results that show the effectiveness of this model, and we summarize and conclude in section 6 .

\section{The 4D tabular macromodel}

We have previously presented a 4-dimensional (4D) table-based macromodel in $[10,11,13]$ for combinational circuits. In this paper, we will show that the method extends to sequential circuits; we will also show that the table can be accurately represented by a simple analytical equation, and we will describe the required automatic characterization flow. The 4D macromodel considers the average power of a circuit to be a function of four variables:

$$
P_{\text {avg }}=f\left(P_{\text {in }}, D_{\text {in }}, S C_{\text {in }}, D_{\text {out }}\right)
$$

These four variables were defined above, but $S C_{i n}$ deserves a few more words. $S C_{i n}$ is equal to the average of all the pair-wise spatial correlations $S C_{i j}$ between signals $x_{i}$ and $x_{j}$, where:

$$
S C_{i j}=\mathcal{P}\left\{x_{i} \wedge x_{j}=1\right\}
$$

i.e., it is the probability of both inputs being high simultaneously. Even though the joint probability is not what is usually referred to as "correlation coefficient" between two random variables, in this case of Boolean variables the joint probability of two bits is enough to capture their complete joint distribution and, therefore, suffices as a measure of their correlation. These four variables are not independent. Indeed, it was observed in $[11,13]$ that $P_{i n}, D_{i n}$ and $S C_{i n}$ should satisfy the following constraints:

$$
\frac{D_{i n}}{2} \leq P_{i n} \leq 1-\frac{D_{i n}}{2}
$$




$$
\frac{n P_{i n}^{2}-P_{i n}}{(n-1)} \leq S C_{i n} \leq P_{i n}
$$

where $n$ is the number of primary inputs.

The combined scatter plot of all ISCAS-85 circuits [14] showing the accuracy of the 4D tabular approach while estimating the total power in presence of correlated input vectors is shown in Fig. 1. The plot reports normalized power values, so that the results for all the circuits can be examined on the same plot. The results are summarized in Table 1. The average error in all cases is less than $10 \%$, which shows the accuracy of this $4 \mathrm{D}$ tabular approach.

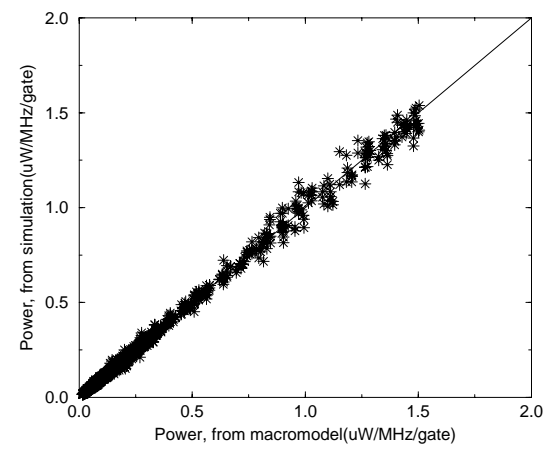

Figure 1. Power comparison between correlated input vector stream and $4 \mathrm{D}$ macromodel, when total power is estimated.

\section{Extension to sequential circuits}

All previous work in this area was restricted to combinational circuits. In this section we will show that our macromodel extends with ease to sequential circuits. Upon first consideration, it would seem that primary inputs information is not sufficient to model the power of sequential circuits, and that some information on the state bits would have to be required. However, when the objective is to estimate the average power, the fact that the state over a long time period becomes independent of the initial state leads to significant simplification of the problem. Indeed, it was shown in [15], that if the sequence of inputs to a sequential machine is of order $k$, then a lag$k$ Markov chain correctly models the input sequence as well as the $k$-step conditional probabilities of the primary primary inputs and internal states.

As a result, if the input signal temporal correlations die down with time, which is a reasonable assumption in practice, then under steady state the state bits distribution is completely determined by the primary inputs distribution, and the statistics of the primary inputs should be sufficient to model the power of a sequential circuits. Indeed, we have experimentally found out that the same 4D macromodel also works for sequential circuits, as will be shown in the experimental results section below.

\section{Equation-based macromodel}

The table-based macromodel requires a lot of memory for storing the table and also requires a lot of time to build the whole table. We have developed an alternate approach by which we can fit a general non-linear equation to the function $f(\cdot)$ in (1) without user intervention and with much less time than it takes to fill the table. This general equation is fixed and is used as the starting point for all circuits - no user intervention is required in the choice of equation. We will refer to this equation as the template. This works because, even though the function $f(\cdot)$ is non-linear, it turns out that in practice it is "not too non-linear" to defy fitting, and a general polynomial template turns out to be sufficient.

For efficiency reasons, one would like to use the lowest order polynomial template that works. One option is the linear function:

$$
\hat{P}_{\text {avg }}=c_{0}+c_{1} P_{\text {in }}+c_{2} D_{\text {in }}+c_{3} S C_{\text {in }}+c_{4} D_{\text {out }}
$$

where the coefficients $c_{i}$ are unknown and are to be determined during the characterization using regression analysis. To estimate the regression variables $c_{i}$, we generated 1000 blocks of correlated vector streams for different values of $P_{i n}, D_{i n}$, and $S C_{i n}$, covering a wide range of input statistics. For each block of vectors, Monte Carlo simulation [16] was used to estimate the average power and to compute the value of $D_{\text {out }}$. Using this set of data and the standard linear least squares method [17], the regression variables $c_{i}$ were estimated. 
To test the accuracy of the fit, we again generated 1000 blocks of correlated input vectors for different values of $P_{i n}, D_{i n}$, and $S C_{i n}$. Using Monte Carlo simulation [16], the average power $\left(P_{\text {avg }}\right)$ and $D_{\text {out }}$ were estimated. For the given $P_{i n}, D_{i n}, S C_{i n}$, and $D_{\text {out }}$ values, the average power $\hat{P}_{a v g}$ was then found using (5) and the relative error between $P_{a v g}$ and $\hat{P}_{a v g}$ was computed and is shown in Table 2. It is evident from the table that a linear function is not good enough for estimating the average power for most of the circuits.

Table 1. Error in the 4D approach, while estimating total power

\begin{tabular}{|c|c|c|}
\hline Circuit & Average Error & Max.Error \\
\hline c432 & $5.56 \%$ & $-27.8 \%$ \\
\hline c880 & $6.7 \%$ & $50.61 \%$ \\
\hline c1908 & $3.85 \%$ & $31.17 \%$ \\
\hline c2670 & $7.8 \%$ & $37.53 \%$ \\
\hline c3540 & $7.5 \%$ & $44.19 \%$ \\
\hline c5315 & $3.48 \%$ & $29.03 \%$ \\
\hline c6288 & $9.6 \%$ & $43.15 \%$ \\
\hline c7552 & $8.95 \%$ & $-45.79 \%$ \\
\hline c499 & $5.96 \%$ & $46.3 \%$ \\
\hline c1355 & $6.19 \%$ & $33.56 \%$ \\
\hline
\end{tabular}

Table 2. Average and maximum error when total power was estimated using linear function

\begin{tabular}{|c|c|c|}
\hline Circuit & Avg. Error & Max.Error \\
\hline c499 & $36.6 \%$ & $1407.2 \%$ \\
\hline c880 & $19.7 \%$ & $257.16 \%$ \\
\hline c1355 & $34.7 \%$ & $993.9 \%$ \\
\hline c1908 & $11.6 \%$ & $68.18 \%$ \\
\hline c432 & $11.01 \%$ & $30.45 \%$ \\
\hline c5315 & $11.4 \%$ & $89.84 \%$ \\
\hline c2670 & $20.36 \%$ & $304.6 \%$ \\
\hline c3540 & $13.07 \%$ & $258.6 \%$ \\
\hline c7552 & $15.5 \%$ & $85.8 \%$ \\
\hline c6288 & $31.9 \%$ & $257.16 \%$ \\
\hline
\end{tabular}

To improve the accuracy, another option is the quadratic function:

$$
\begin{aligned}
\hat{P}_{a v g} & =c_{0}+c_{1} P_{i n}+c_{2} D_{i n}+c_{3} S C_{i n}+c_{4} D_{\text {out }} \\
& +c_{5} P_{i n} D_{i n}+c_{6} P_{i n} S C_{i n}+c_{7} P_{i n} D_{\text {out }}+c_{8} D_{i n} S C_{i n}+c_{9} D_{\text {in }} D_{\text {out }}+c_{10} S C_{\text {in }} D_{\text {out }} \\
& +c_{11} P_{\text {in }}^{2}+c_{12} D_{\text {in }}^{2}+c_{13} S C_{\text {in }}^{2}+c_{14} D_{\text {out }}^{2}
\end{aligned}
$$

Using the same approach as above, the regression variables were estimated and the accuracy of the results was tested and is shown in Table 3 . It is evident from the table that the quadratic function is better for estimating the average power for most of the circuits except c499 and c1355 for which the average error was very high, above $15 \%$.

We also investigated the general cubic form. Due to space limitations, we are not showing the cubic equation here as it consists of 35 coefficients. Table 4 shows the average error and maximum error for the case of a cubic. It is clear that the improvement in the error is not much for most of the circuits, except for c499 and c1355 for which the quadratic function did not do well. This observation was found to hold in general, that for many circuits the quadratic model is enough, but the cubic model can still be superior in some cases. As a result, we use a hybrid approach by which we start with the quadratic model by default and increase the order of the model to a cubic only if the measured error during characterization is too big. It was observed that the cubic function was the highest order function required by all the ISCAS-85 circuits. Similar experiments were performed on sequential circuits, except that the power was estimated using [18]. It was observed that the quadratic function is sufficient for all the sequential circuits that we tested.

Table 3. Average and maximum error when total power was estimated using the quadratic model

\begin{tabular}{|c|c|c|}
\hline Circuit & Avg. Error & Max.Error \\
\hline c499 & $25.64 \%$ & $1319.5 \%$ \\
\hline c880 & $5.9 \%$ & $48.74 \%$ \\
\hline c1355 & $18.08 \%$ & $107.9 \%$ \\
\hline c1908 & $4.9 \%$ & $57.74 \%$ \\
\hline c432 & $4.19 \%$ & $36.8 \%$ \\
\hline c5315 & $3.6 \%$ & $32.56 \%$ \\
\hline c2670 & $10.25 \%$ & $42.25 \%$ \\
\hline c3540 & $5.86 \%$ & $50.49 \%$ \\
\hline c7552 & $8.8 \%$ & $50.48 \%$ \\
\hline c6288 & $9.6 \%$ & $55.74 \%$ \\
\hline
\end{tabular}

Table 4. Average and maximum error when total power was estimated using the cubic model

\begin{tabular}{|c|c|c|}
\hline Circuit & Avg. Error & Max.Error \\
\hline c499 & $8.3 \%$ & $56.06 \%$ \\
\hline c880 & $5.2 \% \%$ & $45.41 \%$ \\
\hline c1355 & $12.07 \%$ & $50.78 \%$ \\
\hline c1908 & $4.12 \%$ & $34.31 \%$ \\
\hline c432 & $3.13 \%$ & $24.16 \%$ \\
\hline c5315 & $3.8 \%$ & $40.42 \%$ \\
\hline c2670 & $6.25 \%$ & $39.45 \%$ \\
\hline c3540 & $3.7 \%$ & $46.38 \%$ \\
\hline c7552 & $5.6 \%$ & $47.96 \%$ \\
\hline c6288 & $8.3 \%$ & $54.6 \%$ \\
\hline
\end{tabular}

\section{Characterization}

To derive the analytical expressions discussed above, we have developed an automatic characterization process using the recursive least squares (RLS) [19] algorithm. RLS is used in adaptive 
filtering for on-line estimation of filter parameters. The following summary of the general RLS algorithm is given for convenience.

\subsection{RLS algorithm}

Let $y=f\left(x_{1}, x_{2}, \ldots, x_{p}\right)$ be a real valued function of real variables. We will use bold font to denote vector or matrix quantities. Let the (column) vector $\mathbf{x}$ be the vector of the $p$ variables, so that the transpose of $\mathbf{x}$ is the (row) vector $\mathbf{x}^{T}=\left[\begin{array}{llll}x_{1} & x_{2} & \cdots & x_{p}\end{array}\right]$ and we write $y=f(\mathbf{x})$. We are interested in approximating $f(\cdot)$ with a closed form analytical expression $\hat{y}=\mathbf{c}^{T} \mathbf{u}$, where $\mathbf{c}^{T}=\left[\begin{array}{lllll}c_{0} & c_{1} & \cdots & c_{m-1}\end{array}\right]$ is a vector of constant coefficients whose values are to be determined and $\mathbf{u}^{T}=\left[\begin{array}{llll}u_{0} & u_{1} & \cdots & u_{m-1}\end{array}\right]$, where each $u_{i}$ is some function of the variables $x_{1}, x_{2}, \ldots, x_{p}$. We would like to find a vector $\mathbf{c}$ so that $\hat{y}$ is a good approximation to $y$.

To this end, suppose we generate $n$ randomly chosen samples $\mathbf{x}(1), \mathbf{x}(2), \ldots, \mathbf{x}(n)$, from which we also compute the corresponding $y(i)$, for $i=1,2, \ldots, n$. Consider the error $e(i)=y(i)-\hat{y}(i)$ and the cumulative error $\zeta(n)=\sum_{i=1}^{n}|e(i)|^{2}$. One way of finding an appropriate $\mathbf{c}$ is to find one that minimizes the error $\zeta(n)$. Typically, the solution will depend on $n$, and we denote it by $\hat{\mathbf{c}}(n)$. Finding such a $\hat{\mathbf{c}}(n)$ is the traditional problem of least-squares fitting which is solved using standard linear regression techniques [17]. The result is that $\hat{\mathbf{c}}(n)$ can be obtained as the solution to the following system of linear equations:

$$
\boldsymbol{\Phi}(n) \hat{\mathbf{c}}(n)=\mathbf{z}(n)
$$

where $\boldsymbol{\Phi}(n)$ is a $m \times m$ matrix, and $\mathbf{z}(n)$ is a $m \times 1$ vector, given by:

$$
\mathbf{\Phi}(n)=\sum_{i=1}^{n} \mathbf{u}(i) \mathbf{u}^{T}(i) \quad \text { and } \quad \mathbf{z}(n)=\sum_{i=1}^{n} \mathbf{u}(i) y(i)
$$

The solution $\hat{\mathbf{c}}(n)=\boldsymbol{\Phi}^{-1}(n) \mathbf{z}(n)$ is the coefficient vector that minimizes the error $\zeta(n)$ over the observed $n$ samples. In order for $\hat{y}$ to be a good approximation for $y$ over the whole domain of $f(\cdot)$, we need to use a large number $n$ of samples. It would be desirable to iteratively add more samples while monitoring the error, but this requires inverting $\boldsymbol{\Phi}(n)$ every time. In the RLS algorithm, this problem is overcome by using the matrix inversion lemma which leads to an iterative update mechanism for $\boldsymbol{\Phi}^{-1}(n)$ that does not require any matrix inversions [19], as follows:

1. Initialize $\boldsymbol{\Phi}^{-1}(0)$ and $\hat{\mathbf{c}}(0)$

2. For $n=1,2, \ldots$, until converged, do:

a. Compute the $m \times 1$ gain vector $\mathbf{k}(n)$ as follows:

$$
\mathbf{k}(n)=\frac{\boldsymbol{\Phi}^{-1}(n-1) \mathbf{u}(n)}{1+\mathbf{u}^{T}(n) \boldsymbol{\Phi}^{-1}(n-1) \mathbf{u}(n)}
$$

b. Update the coefficient vector $\hat{\mathbf{c}}(n)=\hat{\mathbf{c}}(n-1)+\mathbf{k}(n)\left[y(n)-\hat{\mathbf{c}}^{T}(n-1) \mathbf{u}(n)\right]$ 3. End.

c. Update the correlation matrix $\boldsymbol{\Phi}^{-1}(n)=\left[\mathbf{I}-\mathbf{k}(n) \mathbf{u}^{T}(n)\right] \mathbf{\Phi}^{-1}(n-1)$

To use the above algorithm, the initial values $\Phi(0)$ and $\hat{c}(0)$ are required. A common method of initialization is to use:

$$
\begin{gathered}
\boldsymbol{\Phi}(0)=\sum_{i=1}^{n_{0}} \mathbf{u}(i) \mathbf{u}^{T}(i) \\
\hat{\mathbf{c}}(0)=\boldsymbol{\Phi}^{-1}(0) \sum_{i=1}^{n_{0}} \mathbf{u}(i) y(i)
\end{gathered}
$$

where $n_{0}$ data points have been accumulated before starting the RLS algorithm.

In our case $y=P_{\text {avg }}, \mathbf{u}^{T}=\left[P_{i n}^{k}, D_{i n}^{k}, \cdots, 1\right]$ and $\mathbf{c}^{T}=\left[c_{m-1}, \cdots, c_{0}\right]$, where $k$ is the order of the analytical function and $m$ is the number of coefficients to be estimated. We compute $P_{a v g}$ for any given combination of the signal statistics $P_{i n}, D_{i n}$, and $S C_{i n}$ by using Monte Carlo power estimation techniques [16] (for combinational circuits) and [18] (for sequential circuits). Convergence of the RLS algorithm is guaranteed if the excitation is persistent [19]. In other words convergence is guaranteed if the data points at the different time instants are independent 
of each other. This is guaranteed in our case as $P_{i n}, D_{i n}$ and $S C_{i n}$ values are chosen randomly from the feasible region given by (3) and (4).

\subsection{Convergence}

The RLS algorithm is standard textbook material, which we have applied to the power macromodeling problem. However, the convergence criterion to be used to stop the iterative updates depends on the particular application. In this section, we describe a novel method of stopping the RLS iterations that is useful for power macromodeling.

Since all we care about is the accuracy of the predicted value $\hat{y}$ and how well it approximates $y$, we need not wait for all the components of the coefficient vector $\hat{\mathbf{c}}(n)$ to converge. Instead, some norm of $\hat{\mathbf{c}}(n)$ may suffice. Instead of using an arbitrary norm (which we have found can require a large number of iterations), a more efficient and more meaningful method of checking convergence, which indirectly monitors some aggregate measure of convergence of $\hat{\mathbf{c}}(n)$, is to do the following. Consider the following relative error terms, for $i=1,2, \ldots, n$ :

$$
r^{(n)}(i)=\frac{\left|y(i)-\hat{\mathbf{c}}^{T}(n) \mathbf{u}(i)\right|}{y(i)}
$$

If the $\mathbf{x}(i)$ are iid (independent and identically distributed) random vectors, then the $\mathbf{u}(i)$ and $y(i)$ are also iid. For a given fixed $n$, it also follows that the $r^{(n)}(i)$ are also iid, so that the mean of each $r^{(n)}(i)$ is independent of $i$, and depends only on $n$, so that we denote it by:

$$
\mu_{n}=E\left[r^{(n)}(i)\right]
$$

where $E[\cdot]$ is the expected value (or mean) operator. As a side note, notice that $r^{(i)}(i)$ are not iid, because $\hat{\mathbf{c}}(i)$ depends on previous samples. If we now define:

$$
r_{n}=\frac{1}{n} \sum_{i=1}^{n} r^{(n)}(i)
$$

then it follows, under very general conditions of ergodicity, that:

$$
\lim _{n \rightarrow \infty}\left|r_{n}-\mu_{n}\right|=0
$$

so that, at some point, the known (measured) sample mean $r_{n}$ converges to the unknown true mean $\mu_{n}$. Standard methods of mean estimation in statistics (Monte Carlo Mean Estimation [20] [16]) can be used to check if this convergence has been achieved to within some (user-specified) accuracy, with a certain amount of (user-specified) confidence. Once this has been achieved, we start to use $r_{n}$ as an estimate of $\mu_{n}$, which we consider to be a measure of the error of the model. We consider the model to be "good enough" when $\mu_{n}$ is small enough.

In our implementation, we start with the quadratic model and apply RLS while monitoring the convergence of $r_{n}$ to $\mu_{n}$. Once that has been achieved, then if $\mu_{n}$ is below some user-specified error threshold $\mathcal{E}$, we stop the algorithm. Otherwise, we continue to iterate while monitoring $\mu_{n}$ and declare convergence if it goes below $\mathcal{E}$. If we reach a point where $\mu_{n}$ has leveled off at some value larger than $\mathcal{E}$, we switch over to the cubic model and re-evaluate the error and continue to iterate if needed. The process is terminated once either $\mu_{n}<\mathcal{E}$ or if it levels off again at some value larger than $\mathcal{E}$. The experimental results to be given in the next section are based on a setting of $\mathcal{E}=10 \%$.

\section{Results}

In this section, we report the results of our equation-based power macromodeling approach on the ISCAS-85 and ISCAS-89 circuits. The ISCAS-85 circuits are combinational, and the ISCAS-89 circuits are sequential. We have implemented this approach and built the power macromodels for a number of combinational and sequential circuits. First we will discuss the accuracy for the case of the combinational circuits and then for the sequential circuits. All the results below are based on an error threshold setting of $\mathcal{E}=10 \%$ (for convergence of (15)), an error tolerance of $\epsilon=5 \%$ and a confidence of $(1-\alpha)=95 \%$ (for the Monte Carlo mean estimation).

We start by randomly generating blocks of input vectors for various values of $P_{i n}, D_{i n}$, and $S C_{i n}$ that satisfy (3) and (4), using the approach documented in [11, 13]. A total of 1000 


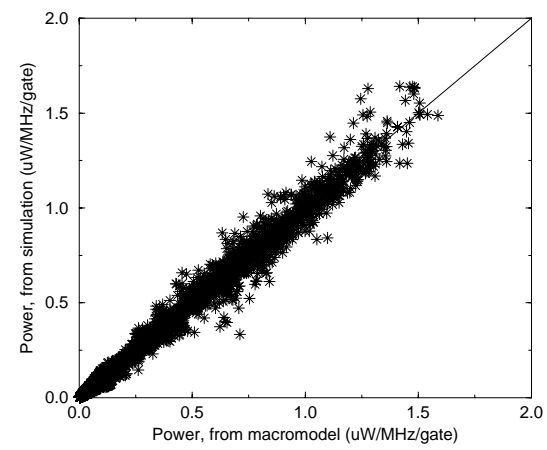

Figure 2. Power comparisons for the ISCAS-85 circuits.

such blocks were generated for every circuit, for which power was estimated using Monte Carlo simulation [16], based on a gate-level simulation with a scalable-delay gate timing model. The Monte Carlo simulation also provides accurate estimation of $D_{\text {out }}$. The power values predicted by the analytical function were compared to those from the gate-level Monte Carlo simulation, and the results are shown in Fig. 2. The fit is very good and shows that it is indeed possible to do high-level power modeling across the whole range of input switching statistics.

For a more detailed comparison, three different error measures were computed for every circuit: the average absolute value of the relative error, the maximum absolute value of the relative error, and the standard deviation of the absolute value of the relative error. The results are summarized in Table 5, which shows, for each circuit, the number of inputs (\#I), outputs $(\# \mathrm{O})$, and gates $(\# \mathrm{G})$, the model finally used by RLS, the time taken by RLS to build the model, the different error measures, and the number of RLS iterations (\#RLS) required to build the model. It is clear that the quadratic model suffices for most circuits. Also the average error for most of the circuits is less than $10 \%$. Even though the maximum error is high in some circuits, it was observed that the number of sample points with those error values was very small, as can be seen from the small values of standard deviation and from the good fit in Fig. 2. Also, it is clear from Fig. 2 that the cases of high relative error shown in the table must correspond to really small power values (in the lower left corner of the figure), because, except for maybe 4 points out of 10,000, the data shown in the figure shows small values of relative error.

Table 5. Accuracy of the equation-based macromodel on the ISCAS- 85 circuits.

Error statistics are from 1000 tests, using different values of input switching statistics. Under the Model column, C means Cubic and Q means Quadratic. Execution times are on a SUN Ultra Sparc 1 with $64 \mathrm{MB}$ of RAM.

\begin{tabular}{|c|c|c|c|c|c|c|c|c|c|}
\hline Circuit & $\# \mathrm{I}$ & $\# \mathrm{O}$ & $\# \mathrm{G}$ & Model & Time & Avg. Error & Max. Error & Std. Dev. & \#RLS \\
\hline $\mathrm{c} 499$ & 41 & 32 & 202 & $\mathrm{C}$ & $16.31 \mathrm{~m}$ & $12.03 \%$ & $48.5 \%$ & $10.19 \%$ & 258 \\
\hline $\mathrm{c} 880$ & 60 & 26 & 383 & $\mathrm{Q}$ & $29.10 \mathrm{~m}$ & $6.4 \%$ & $53.88 \%$ & $6.79 \%$ & 162 \\
\hline $\mathrm{c} 1355$ & 41 & 32 & 546 & $\mathrm{C}$ & $22.2 \mathrm{~m}$ & $13.06 \%$ & $53.98 \%$ & $10.39 \%$ & 230 \\
\hline $\mathrm{c} 1908$ & 33 & 25 & 880 & $\mathrm{Q}$ & $36.14 \mathrm{~m}$ & $4.66 \%$ & $30.39 \%$ & $4.18 \%$ & 120 \\
\hline $\mathrm{c} 432$ & 36 & 7 & 160 & $\mathrm{Q}$ & $22.52 \mathrm{~m}$ & $3.46 \%$ & $25.75 \%$ & $3.35 \%$ & 163 \\
\hline $\mathrm{c} 5315$ & 178 & 123 & 2307 & $\mathrm{Q}$ & $3.74 \mathrm{hrs}$ & $3.53 \%$ & $32.63 \%$ & $3.40 \%$ & 286 \\
\hline $\mathrm{c} 2670$ & 233 & 140 & 1193 & $\mathrm{C}$ & $2.24 \mathrm{hrs}$ & $6.09 \%$ & $46.16 \%$ & $6.55 \%$ & 207 \\
\hline $\mathrm{c} 3540$ & 50 & 22 & 1669 & $\mathrm{Q}$ & $5.08 \mathrm{hrs}$ & $4.66 \%$ & $40.26 \%$ & $5.24 \%$ & 228 \\
\hline $\mathrm{c} 7552$ & 207 & 108 & 3512 & $\mathrm{Q}$ & $19.75 \mathrm{hrs}$ & $8.23 \%$ & $46.32 \%$ & $6.65 \%$ & 601 \\
\hline $\mathrm{c} 6288$ & 32 & 32 & 2406 & $\mathrm{C}$ & $9.23 \mathrm{hrs}$ & $9.68 \%$ & $54.03 \%$ & $10.15 \%$ & 286 \\
\hline
\end{tabular}

Similar experiments were carried out for the ISCAS-89 sequential circuits, except that the average power in this case was estimated using [18]. The comparison with gate level power estimation is shown in Fig. 3. Here again, the fit is seen to be very good, even better than in the combinational circuits case. Table 6 reports the detailed results and contains the same columns as table 5, except that the column marked \#FF gives the number of flip-flops in each circuit. 
The quadratic model turned out to be sufficient for all these circuits. The average error is less than $5 \%$ for most circuits, and the standard deviation of the error is very small, which shows the accuracy of our approach.

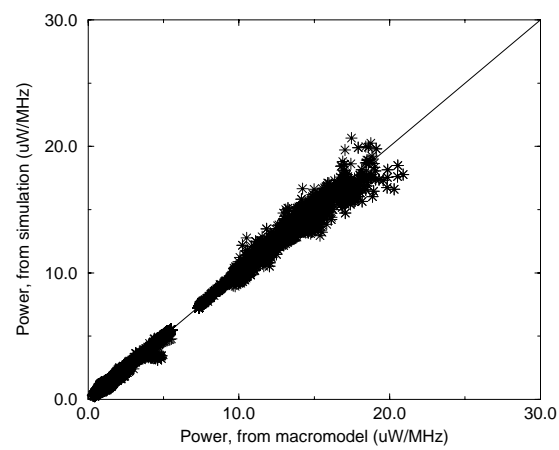

Figure 3. Power comparisons for the ISCAS-89 circuits.

Table 6. Accuracy of the equation-based macromodel on the ISCAS- 89 circuits. Error statistics are from 1000 tests, using different values of input switching statistics. Under the Model column, C means Cubic and Q means Quadratic.

Execution times are on a SUN Ultra Sparc 1 with $64 \mathrm{MB}$ of RAM.

\begin{tabular}{|c|c|c|c|c|c|c|c|c|c|c|}
\hline Circuit & $\# \mathrm{I}$ & $\# \mathrm{O}$ & $\# \mathrm{G}$ & $\# \mathrm{FF}$ & Model & Time & Avg. Error & Max. Error & Std. Dev. & \#RLS \\
\hline s349 & 9 & 11 & 161 & 15 & $\mathrm{Q}$ & $22.36 \mathrm{~m}$ & $7.66 \%$ & $46.31 \%$ & $7.85 \%$ & 201 \\
\hline $\mathrm{s} 344$ & 9 & 11 & 160 & 15 & Q & $19.87 \mathrm{~m}$ & $6.48 \%$ & $38.95 \%$ & $7.59 \%$ & 120 \\
\hline $\mathrm{s} 400$ & 3 & 6 & 164 & 21 & Q & $25.8 \mathrm{~m}$ & $2.65 \%$ & $29.95 \%$ & $2.68 \%$ & 151 \\
\hline s713 & 35 & 23 & 393 & 19 & Q & $49.88 \mathrm{~m}$ & $1.65 \%$ & $9.23 \%$ & $1.52 \%$ & 129 \\
\hline s832 & 18 & 19 & 287 & 6 & Q & $1.85 \mathrm{hrs}$ & $1.39 \%$ & $13.15 \%$ & $1.54 \%$ & 185 \\
\hline s526 & 3 & 6 & 193 & 21 & Q & $31.3 \mathrm{~m}$ & $6.2 \%$ & $42.95 \%$ & $7.57 \%$ & 171 \\
\hline s1494 & 8 & 19 & 647 & 6 & $\mathrm{Q}$ & $1.56 \mathrm{hrs}$ & $5.0 \%$ & $34.1 \%$ & $5.09 \%$ & 189 \\
\hline $\mathrm{s} 1488$ & 8 & 19 & 653 & 6 & $\mathrm{Q}$ & $33.21 \mathrm{~m}$ & $3.65 \%$ & $33.51 \%$ & $3.27 \%$ & 123 \\
\hline $\mathrm{s} 1423$ & 17 & 5 & 657 & 74 & Q & $1.09 \mathrm{hrs}$ & $5.29 \%$ & $19.99 \%$ & $4.54 \%$ & 160 \\
\hline s386 & 7 & 7 & 159 & 6 & $\mathrm{Q}$ & $28.43 \mathrm{~m}$ & $1.49 \%$ & $16.98 \%$ & $1.59 \%$ & 235 \\
\hline $\mathrm{s} 382$ & 3 & 6 & 158 & 21 & $\mathrm{Q}$ & $28.64 \mathrm{~m}$ & $2.56 \%$ & $29.52 \%$ & $3.7 \%$ & 160 \\
\hline $\mathrm{s} 420$ & 18 & 1 & 218 & 16 & Q & $56.96 \mathrm{~m}$ & $0.94 \%$ & $4.49 \%$ & $0.68 \%$ & 164 \\
\hline $\mathrm{s} 641$ & 35 & 24 & 379 & 19 & Q & 1.33hrs & $1.71 \%$ & $8.69 \%$ & $1.22 \%$ & 179 \\
\hline s1196 & 14 & 14 & 529 & 18 & Q & $1.69 \mathrm{hrs}$ & $0.3 \%$ & $3.22 \%$ & $0.43 \%$ & 2716 \\
\hline s510 & 19 & 7 & 211 & 6 & Q & $1.45 \mathrm{hrs}$ & $0.11 \%$ & $0.58 \%$ & $0.1 \%$ & 147 \\
\hline s953 & 16 & 23 & 395 & 29 & Q & $5.5 \mathrm{hrs}$ & $0.21 \%$ & $2.1 \%$ & $0.29 \%$ & 227 \\
\hline $\mathrm{s} 298$ & 3 & 6 & 119 & 14 & Q & $38.46 \mathrm{~m}$ & $2.56 \%$ & $9.97 \%$ & $2.09 \%$ & 288 \\
\hline $\mathrm{s} 1238$ & 14 & 14 & 508 & 18 & $\mathrm{Q}$ & $25.6 \mathrm{~m}$ & $0.58 \%$ & $16.5 \%$ & $2.0 \%$ & 456 \\
\hline $\mathrm{s} 444$ & 3 & 6 & 181 & 21 & Q & $22.2 \mathrm{~m}$ & $2.57 \%$ & $9.99 \%$ & $2.55 \%$ & 137 \\
\hline s820 & 18 & 19 & 289 & 5 & Q & $1.47 \mathrm{hrs}$ & $1.58 \%$ & $9.6 \%$ & $1.63 \%$ & 141 \\
\hline s838 & 34 & 1 & 446 & 32 & Q & $2.86 \mathrm{hrs}$ & $0.98 \%$ & $4.04 \%$ & $0.87 \%$ & 179 \\
\hline s5378 & 35 & 49 & 2779 & 179 & Q & $5.5 \mathrm{hrs}$ & $1.11 \%$ & $5.22 \%$ & $0.77 \%$ & 89 \\
\hline s9234 & 36 & 39 & 5597 & 211 & Q & $14.64 \mathrm{hrs}$ & $2.9 \%$ & $21.2 \%$ & $2.56 \%$ & 127 \\
\hline
\end{tabular}




\section{Conclusion}

Since gate-level power estimation can be time-consuming and because power estimation from a high level of abstraction is desirable so as to reduce design time and cost, we have proposed an equation-based power macromodeling approach for combinational and sequential circuits. Our macromodel consists of an analytical function with four variables: average input signal probability, average input switching activity, average input spatial correlation coefficient, and average output (zero-delay) switching activity. We also presented a Recursive Least Squares (RLS) algorithm by which such an analytical expression can be generated. The proposed model works for all possible signal switching statistics and no user intervention is needed for the model characterization. The only thing that the user has to specify is how much accuracy is desired. The macromodel has been built and tested for many combinational and sequential benchmark circuits.

\section{References}

[1] F. N. Najm, "A survey of power estimation techniques in VLSI circuits," IEEE Transactions on VLSI Systems, pp. 446-455, Dec. 1994.

[2] P. Landman, High-level power estimation, "International Symposium on Low Power Electronics and Design," pp. 29-35, Monterey, CA, August 12-14, 1996.

[3] M. Nemani and F. N. Najm, "Towards a High-Level Power Estimation Capability," IEEE Transactions on CAD, vol. 15 pp. 588-598, June 1996.

[4] D. Marculescu, R. Marculescu and M. Pedram, "Information Theoretic Measures of Energy Consumption at Register Transfer Level," ACM/IEEE International Symposium on Low Power Design, pp. 87-92, April 1995.

[5] S. R. Powell and P. M. Chau, "Estimating Power Dissipation of VLSI signal Processing Chips: The PFA technique," VLSI Signal Processing IV, pp. 250-259, 1990.

[6] P. E. Landman and J. M. Rabaey, "Architectural Power Analysis: The Dual Bit Type Method," IEEE Transactions on VLSI, vol. 3 pp. 173-187 June 1995.

[7] H. Mehta, R. M. Owens and M. J. Irwin, "Energy Characterization based on Clustering," 33rd ACM/IEEE Design Automation Conference, pp. 702-707, June 1996.

[8] A. Raghunathan, S. Dey and N. K. Jha, "Register-Transfer Level Estimation Techniques for Switching Activity and Power Consumption," IEEE International Conference on Computer-Aided Design, pp. 158-165, November 1996.

[9] Q. Qiu, Q. Wu, Chih-S. Ding, and M. Pedram, "Cycle-accurate macro-models for RT-level power analysis," Proc. International Symposium on Low Power Electronics and Design, pp. 125-130, 1997.

[10] S. Gupta and F. N. Najm, "Power Macromodeling for High Level Power Estimation," 34th ACM/IEEE Design Automation Conference, pp. 365-370, June 1997.

[11] S. Gupta and F. N. Najm, "Power Macromodeling for High Level Power Estimation," University of Illinois, Coordinated Science Laboratory, Report \#UILU-ENG-97-2229, September 1997.

[12] F. N. Najm, "Transition Density: A New Measure of Activity in Digital Circuits," IEEE Trans. on CAD, vol. 12, pp. 310-323, Feb. 1993.

[13] S. Gupta and F. N. Najm, "Power Macromodeling for High Level Power Estimation," Submitted to IEEE Transactions on VLSI, 1997.

[14] F. Brglez and H. Fujiwara, "A neutral netlist of 10 combinational benchmark circuits and a target translator in Fortran," IEEE International Symposium on Circuits and Systems, pp. 695-698, June 1985.

[15] D. Marculescu, R. Marculescu, and M. Pedram, "Sequence Compaction for Probabilistic Analysis of Finite-State Machines," 34th ACM/IEEE Design Automation Conference, pp. 12-15, June 1997.

[16] M. Xakellis and F. N. Najm, "Statistical Estimation of the Switching Activity in Digital Circuits," 31st ACM/IEEE Design Automation Conference, pp. 728-733, June 1994.

[17] G. Seber, Linear Regression Analysis. New York: John Wiley \& Sons, 1977.

[18] J. Kozhaya and F. N. Najm, "Accurate power estimation for large sequential circuits," IEEE International Conference on Computer-Aided Design, pp. 448-493, November 1997.

[19] L. Ljung, System Identification: theory for the user. Engelwood Cliffs, NJ: Prentice Hall, 1987.

[20] I. R. Miller, J. E. Freund, and R. Johnson, Probability and Statistics for Engineers, 4th Ed., Englewood Cliffs, NJ: Prentice-Hall Inc., 1990, pp. 210-211. 